\title{
Exciton Diffusion in Bulk Heterojunction Solar Cells: A Kinetic Monte Carlo Study
}

1,*Willian Fábio Radel (PG), ${ }^{1}$ Bernhard Georg Enders Neto (PQ) and ${ }^{2}$ Luiz Antônio Ribeiro Júnior (PQ)

${ }^{1}$ University of Brasília, PPG-CIMA, Campus Planaltina, 73345-010, Brasília, DF, Brazil, ${ }^{2}$ International Center for Condensed Matter Physics, University of Brasília, P.O. Box 04531, 70.919-970, Brasília, DF, Brazil

Keywords: Excitons, Diffusion, Bulk Heterojunctions.

\section{Introduction}

It is well accepted that the exciton delocalization has a strong impact on the performance of organic solar cells [1]. Large exciton delocalization estimates, for instance, have promoted the theory of long-range charge transfer as a mechanism for efficient charge separation [1]. Moreover, the complex nanoscale morphologies present in bulk heterojunction (BHJ) solar cells have been repeatedly shown to have a significant impact on device performance for a number of different donor-acceptor combinations. Here, by using a kinetic monte carlo approach, the exciton diffusion is in BHJ solar cells is theoretically investigated in morphologies that are free of fullerene species, i. e., polymer or small oligomer donor-acceptor interfaces.

\section{Methodology}

Here, Kinetic Monte Carlo (KMC) simulations are employed to understand some important properties in fullerene-free BHJ organic photovoltaic devices. In order to do so, the BHJ morphologies are generated by using the Ising_OPV code [2] and the exciton dynamics is simulated through the Excimontec toolkit [3].

\section{Results}

In the most important result obtained here, our simulations show how the interaction energy affects the tortuosity of the interconnected domains of the BHJ and the resulting exciton transport behavior in $\mathrm{KMC}$ simulations. One of the BHJ morphologies employed in our simulations is represented below. 


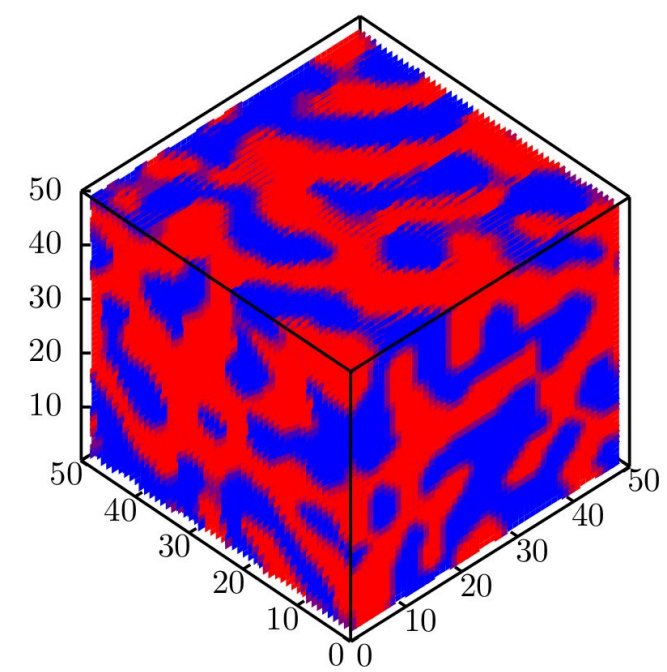

Figure 1: Fullerene-free BHJ morphology of a 50x50x50 nm lattice..

\section{Conclusions}

Overall, we provide a detailed characterization of fullerene-free BHJ morphologies. Furthermore, we investigate the effect of the interaction energy on the exciton diffusion mechanism in these systems. Our results show that the combined approach based in the tolls Ising_OPV and Excimotenc can accurately address the some important optoelectronic properties in model BHJ solar cells.

\section{Acknowledgements}

The authors gratefully acknowledge the financial support from the Brazilian Research Councils CAPES and FAPDF. L.A.R.J. gratefully acknowledges the financial support from the Brazilian Research Council FAPDF grant 0193.000942/2015 and 193.001.511/2017. L.A.R.J. also wishes to thank the Brazilian Ministry of Planning, Budget and Management (Grant DIPLA 005/2016).

\section{References}

[1] J. L. Brédas, et. al. Acc. Chem. Res. 42, 1691-1699, 2009.

[2] M. C. Heiber, et. al. Phys. Rev. App. 2, 014008, 2014.

[3] M. C. Heiber and A. Dhinojwala. J. Phys. Chem. C. 117, 21627-21634, 2013. 